\title{
Carotid stent placement procedures
}

\author{
Sean D. LaVine, M.D. \\ Department of Neurosurgery, College of Physicians and Surgeons of Columbia University, \\ New York, New York
}

Large numbers of patients are currently being treated with endovascular alternatives to open surgical procedures. Over the last decade, neurosurgery has been exposed to this surgical evolution, particularly in the area of coil embolization of cerebral aneurysms. Several lessons have been learned; possibly the most important one is that high-quality scientific study of this new technology is extremely difficult and has not been able to keep pace with the explosion in device advancements.

The next wave of influence in our field is currently being experienced in the treatment of carotid artery (CA) stenosis. Carotid endarterectomy has long been considered the standard of care for a very well-defined patient population with CA stenosis. Despite limited findings in the literature to support such a shift, many patients undergo stent-assisted carotid angioplasty as a true alternative to endarterectomy rather than being selected for endovascular treatment based on objective criteria. The influence of medical device companies has been considerable in this area, and to date, most peer-reviewed data have been generated by company-sponsored trials.

This issue of Neurosurgical Focus will provide the reader with a critical analysis of the current status of carotid angioplasty and stent placement procedures. An area of particular controversy is the need for embolic protection devices during these procedures. Kadkhodayan and his colleagues from the Mallinckrodt Institute summarize their experience with "unprotected" procedures and offer an interesting perspective on the possibility of identifying certain patient groups that are at risk for embolic intraprocedure events. Das, et al., and Levy, et al., summarize the results of current stent trials, and their reviews are particularly timely, considering the recent publication of the Stenting and Angioplasty with Protection in Patients at High Risk for Endarterectomy Trial. ${ }^{1}$ This is one of the few randomized studies in which endarterectomy is com- pared with carotid angioplasty and stent placement performed using a cerebral protection device that will soon be approved by the Food and Drug Administration. Gupta, et al., review the phenomenon of hemodynamic instability after carotid angioplasty and stent placement, with a discussion of the pathophysiological features and the need for intraprocedure pacemaker devices.

The trend in minimally invasive procedures is to improve the technology continually, enabling safer and more effective intervention. In neuroendovascular surgery, this equates to smaller profile, more flexible, less traumatic devices. This allows navigation beyond the cervical CA into intracranial locations. Komotar, et al., present a pair of papers covering the natural history of intracranial CA atherosclerosis and the endovascular treatment options for this serious condition.

Finally, Koebbe, et al., and Ecker, et al., review selected groups of patients, those with recurrent and radiationinduced stenosis, who appear to be better suited for endovascular than for open surgical treatment. It is clear that carotid angioplasty and stent placement will have a major role in the treatment of CA stenosis. Many major centers have already seen a significant impact on case volume, despite limited numbers of conclusive investigations, and researchers conducting randomized trials are having difficulty with recruitment.

\section{Acknowledgments}

Drs. Philip Meyers and Peter Rasmussen provided invaluable assistance with their critical review of the submitted manuscripts.

\section{Reference}

1. Yadav JS, Wholey MH, Kuntz RE, et al: Protected carotidartery stenting versus endarterectomy in high-risk patients. $\mathbf{N}$ Engl J Med 351:1493-1501, 2004. 